University of Nebraska - Lincoln

DigitalCommons@University of Nebraska - Lincoln

2012

\title{
Hysteresis and relaxation in granular permanent magnets
}

\author{
Ralph Skomski \\ University of Nebraska-Lincoln, rskomski2@unl.edu \\ Balamurugan Balamurugan \\ University of Nebraska-Lincoln, balamurugan@unl.edu \\ Thomas A. George \\ University of Nebraska-Lincoln, tgeorge1@unl.edu \\ Mircea Chipara \\ University of Texas Pan American, mchipara@utpa.edu \\ Xiao-Hui Wei \\ University of Nebraska-Lincoln \\ See next page for additional authors
}

Follow this and additional works at: https://digitalcommons.unl.edu/physicssellmyer

Part of the Physics Commons

Skomski, Ralph; Balamurugan, Balamurugan; George, Thomas A.; Chipara, Mircea; Wei, Xiao-Hui; Shield, Jeffrey E.; and Sellmyer, David J., "Hysteresis and relaxation in granular permanent magnets" (2012). David Sellmyer Publications. 229.

https://digitalcommons.unl.edu/physicssellmyer/229

This Article is brought to you for free and open access by the Research Papers in Physics and Astronomy at DigitalCommons@University of Nebraska - Lincoln. It has been accepted for inclusion in David Sellmyer Publications by an authorized administrator of DigitalCommons@University of Nebraska - Lincoln. 


\section{Authors}

Ralph Skomski, Balamurugan Balamurugan, Thomas A. George, Mircea Chipara, Xiao-Hui Wei, Jeffrey E. Shield, and David J. Sellmyer 


\title{
Hysteresis and relaxation in granular permanent magnets
}

\author{
Ralph Skomski, ${ }^{1}$ B. Balamurugan, ${ }^{1}$ Tom A. George,,${ }^{1}$ Mircea Chipara, ${ }^{2}$ Xiao-Hui Wei, ${ }^{1}$ \\ Jeff E. Shield, ${ }^{3}$ and D. J. Sellmyer ${ }^{1}$ \\ ${ }^{1}$ Department of Physics and Astronomy and NCMN, University of Nebraska, Lincoln, Nebraska 68588, USA \\ ${ }^{2}$ Department of Physics and Geology, University of Texas-Pan American, Edinburg, Texas 78539, USA \\ ${ }^{3}$ Department of Mechanical and Materials Engineering and NCMN, University of Nebraska, Lincoln, \\ Nebraska 68588, USA
}

(Presented 31 October 2011; received 24 September 2011; accepted 27 October 2011; published online 24 February 2012)

\begin{abstract}
Some nontrivial aspects of the magnetic and structural characterization of hard-magnetic nanoparticles are investigated. Dilute ensembles are well-described by mean-field theory, although there is an asymmetry between exchange and magnetostatic interaction fields. Corrections to the mean-field approximation are caused by cooperative effects and have the character of Onsager reaction fields, which are much stronger in micromagnetism than in atomic-scale magnetism. The slow dynamics of zero-field-cooled (ZFC) magnetization curves is strongly affected by the particles' magnetic anisotropy, which reduces the corresponding energy-barrier height from 25 to $19.1 k_{\mathrm{B}} T$. ( 2012 American Institute of Physics. [doi:10.1063/1.3672845]
\end{abstract}

\section{INTRODUCTION}

Hard-magnetic nanoparticles, such as $\mathrm{FePt}^{1}$ and $\mathrm{YCo}_{5}{ }^{2}$ can be interpreted as ensembles of interacting magnetic grains, and the same is true for granular permanent magnets. The interactions have far-reaching consequences for hysteresis and for slow magnetization dynamics, as observed in magnetic viscosity and ZFC/FC measurements. The simplest approach is the mean-field approximation, which is also the basis for remanence plots, such as $\Delta m$ plots. However, the mean-field approximation is a poor approach to coercivity and hysteresis and incorrectly treats exchange and magnetostatic interactions on equal footing, describing them as positive and negative interactions, respectively. ${ }^{3,4}$

In this paper, we discuss several theoretical aspects of the magnetism of particulate nanomagnets and discuss how experimental investigations are affected by these theoretical considerations.

\section{PACKING FRACTION AND INTERACTIONS}

Clusters are usually embedded in a matrix material to make a nanocomposite film, and a multilayer structure with alternating cluster layer and matrix layer is usually adopted. Figure 1 shows typical clusters deposited on a TEM grid. The number of particles is conveniently parameterized by the nominal film thickness $t_{\text {nom }}=4 \pi R^{3} N / 3 A$, where $N$ is the total number of particles, $R$ is the particle radius, and $A$ is the film area. For sufficiently dilute particle ensembles with random lateral distribution, the areal coverage $f_{\mathrm{A}}=3 t_{\text {nom }} / 4 R$. This result is independent of whether the particles are truly randomly located or form layered structures, separated by nonmagnetic spacer layers.

For small cluster concentrations, the above equation is quite accurate. In one explicit example, the predicted and measured $f_{\mathrm{A}}$ values were $11 \%$ and $10 \%$, respectively. As the nominal thickness increases, the particles overlap in the twodimensional TEM view, and interactions cause the formation of elongated islands and other structures. The overlap effect can be calculated with the method outlined in ${ }^{5}$ and yields the areal coverage

$$
f_{\mathrm{A}}=1-\exp \left(-3 t_{\mathrm{nom}} / 4 R\right),
$$

where the exponential dependence reflects the randomness of the lateral particle positions. Interactions are more difficult to treat than one might expect from Fig. 1, because $\mathrm{MnAu}$ is essentially antiferromagnetic and the magnetostatic interactions due to the residual moment of the particles are not very strong.

\section{HYSTERESIS-LOOP SLOPE}

Hysteresis loops are a rich source of information on particulate magnets. Our focus is on the loop slope or "micromagnetic susceptibility" $\chi=\mathrm{d} M / \mathrm{d} H$ measured at $H_{c}$. (In magnetic recording, this parameter is referred to as $\alpha$.) The micromagnetic susceptibility $\chi_{0}$ of noninteracting particles is determined by the switching-field distribution $P(H)$, which reflects real-structure features such as particle shape and size distribution. The relation is $\chi_{\mathrm{o}}(H)=2 M_{\mathrm{s}} P(H)$, where $2 M_{s}$ is the magnetization change as the field increases from $-\infty$ to $+\infty$.

A simple mean-field approach is to add an interaction field $\lambda M$ to the external field, which yields the linearized equation $M=\chi_{\mathrm{o}}(H+\lambda M)$ and

$$
\chi=\frac{\chi_{\mathrm{o}}}{1-\chi_{\mathrm{o}} \lambda} .
$$

This micromagnetic susceptibility is structurally and physically very similar to the Stoner susceptibility $\chi=\chi_{0} /[1-I$ $\left.D\left(E_{\mathrm{F}}\right)\right]$. In both cases, the susceptibility $\chi_{\mathrm{o}}$ of a noninteracting system is enhanced by a mean-field type interaction, and in both cases, $\chi$ is overestimated. The Stoner theory sometimes incorrectly predicts ferromagnetism for antiferromagnets, and 


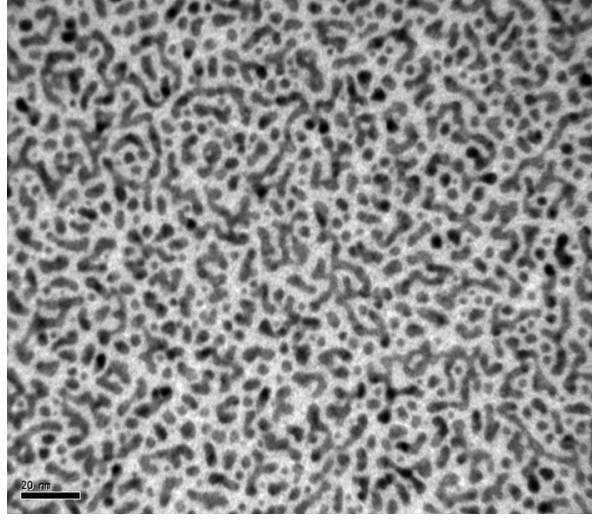

FIG. 1. $L 1_{0}$-ordered MnAu clusters on a TEM grid. The particle agglomeration visible in this image is usually a combination of projection and physical interaction effects.

Eq. (2) breaks down for very high interaction strengths. Note that atomic-scale exchange parameters (or molecular fields) $\lambda \sim J$ often exceed $100 \mathrm{~T}$, as compared to typical switchingfield distribution of the order of $1 \mathrm{~T}$. In fact, when the exchange interaction exceeds a certain threshold, then the particles can be considered as strongly coupled and the excess exchange has very little effect on the hysteresis loop. ${ }^{3}$

The corrections to the mean-field susceptibility have the character of an Onsager reaction field. ${ }^{6}$ Figure 2 illustrates the mechanism. Magnetization changes are not realized by the field itself, but by the torque that acts on a given spin (central spin). In strongly interacting systems, Fig. 2(b), the rotation of the central spin initiates the rotation of neighboring spins, which leads to a substantial reduction of the torque acting on the central spin.

The exchange energy between normalized spins $\mathbf{S}_{0}$ and $\mathbf{S}_{1}$ on neighboring sites $\mathbf{R}_{0}$ and $\mathbf{R}_{1}$ scales as $-J \cos \left(\theta_{0}-\theta_{1}\right)$, and subtracting the irrelevant projection onto the central spin yields

$$
J \rightarrow J\left(1-<\mathbf{S}_{0} \cdot \mathbf{S}_{1}>\right)
$$

This expresses the Onsager reaction field in terms of the spin correlations $C=\left\langle\mathbf{S}_{0} \cdot \mathbf{S}_{1}\right\rangle{ }^{8}$ Aside from a trivial conversion factor, $\lambda=J$, so that Eq. (2) can be rewritten as

$$
\chi=\frac{\chi_{\mathrm{o}}}{1-\chi_{\mathrm{o}} J(1-C)}
$$

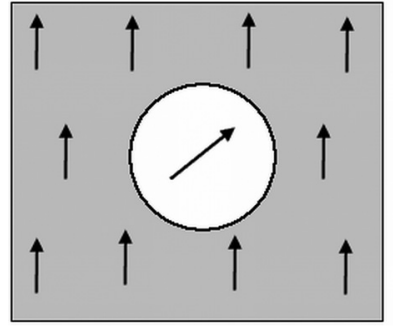

(a)

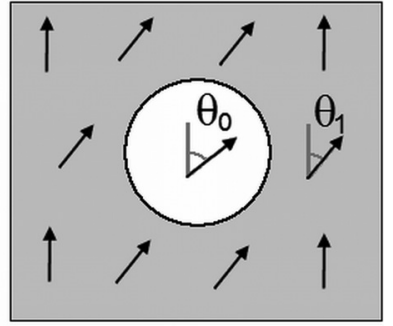

(b)
FIG. 2. Angular correlations in ferromagnets: (a) mean-field model and (b) Onsager correction.

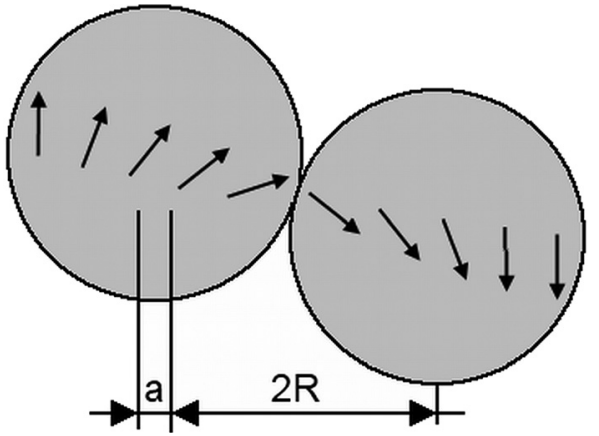

FIG. 3. Nanoscale implementation of the Onsager correction. Onsager correlations in ferromagnets extend over many interatomic distances $a$ but not necessarily over many grain or particle diameters $2 R$.

The Onsager field is usually defined for atomic dipoles, ${ }^{6-8}$ where individual dipoles can rotate, for example, due to thermal excitation. In this case, the Onsager field is a small perturbation with the small parameter $1 / z$, where $z$ is the number of nearest atomic neighbors. ${ }^{7}$ In micromagnetism, neighboring atomic spins are nearly parallel and the Onsager term leads to an almost complete cancellation of the exchange field. On a nanoscale, the interaction may be mean-field like $\left(\chi_{\mathrm{o}} \lambda \ll 1\right)$, of intermediate strength $\left(\chi_{\mathrm{o}} \lambda \sim 1\right)$, or cooperative $\left(\chi_{\mathrm{o}} \lambda \gg 1\right)$. Figure 3 shows the intermediate case, with partial spin correlations.

\section{EXCHANGE AND MAGNETOSTATIC INTERACTIONS}

It is tempting to associate positive and negative values of $\lambda$ (or $J$ ) with exchange and magnetostatic interactions, respectively, as done in Henkel, $\Delta m$, and other remanence plots. However, magnetostatic interactions cannot be mapped onto a negative interaction field $\lambda<0$. This is seen by considering local rather than global mean-field approximations, where the mean field varies across the sample. Magnetostatic interactions favor columnar spins structures with alternating $\uparrow$ and $\downarrow$ particle columns, and the mean field changes sign between neighboring columns. The corresponding mean-field equations have the character of a local mean-field theory. 4,9

Figure 4 shows the behavior of a macroscopically compact (rather than thin-film-like) granular magnet with (a) ferromagnetic interparticle exchange and (b) magnetostatic interactions. Aside from the opposite changes in the loop

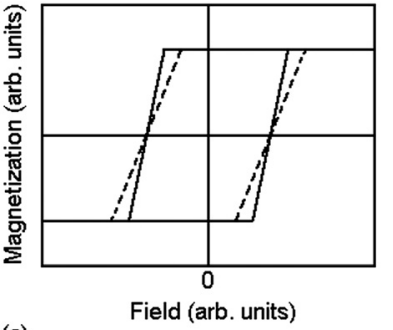

(a)

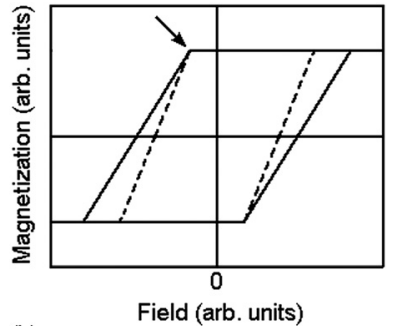

(b)
FIG. 4. Effect of interactions on the hysteresis loop: (a) exchange interactions and (b) magnetostatic interactions. The dashed lines correspond to noninteracting particles and the arrow in (b) indicates where the net magnetostatic interaction is zero. 
slope, there is a "fixed point" [arrow in (b)] where the magnetostatic interactions are not effective. This is because the dipole field created by a cubic or spherical neighborhood of parallel spins is zero. For example, an octahedral environment has 6 nearest neighbors with relative field contributions of 1 (top), 4 times -[1/2] (in-plane neighbors), and 1 (bottom). This is true for any value of the average magnetization, and to obtain a net interaction, one must define two sublattices, so that the interaction-field contributions from the octahedron change to 1, 4 times $+[1 / 2]$, and 1 . The same effect exists in thin films, but there it is less obvious due to the strong demagnetizing field.

\section{SLOW DYNAMICS}

Field- and zero-field-cooled magnetization curves are frequently used to investigate ensembles of magnetic nanoparticles. Here we consider the effect of magnetic anisotropy on the zero-field-cooled (ZFC) peaks of noninteracting hardmagnetic particles in a small field $H$. We assume that all particles are identical and Stoner-Wohlfarth-like, of anisotropy constant $K$ and volume $V$. We further assume that the particles are $c$-axis aligned, which yields the well-known energy barriers

$$
E_{\mathrm{b}}=K V\left(1 \pm H / H_{\mathrm{o}}\right)^{2}
$$

Here $H_{\mathrm{o}}=2 K / \mu_{\mathrm{o}} M$ is the anisotropy field of the particles. The ZFC peak position is obtained by using a constant heating rate $\gamma=\mathrm{d} T / \mathrm{d} t$. The peak temperature $T_{\mathrm{o}}$, which corresponds to a heating time $t_{\mathrm{o}}=T_{\mathrm{o}} / \gamma$, is typically estimated from $K V=\ln \left(\Gamma_{\mathrm{o}} t_{\mathrm{o}}\right) k_{\mathrm{B}} T_{\mathrm{o}} \approx 25 k_{\mathrm{B}} T_{\mathrm{o}}$, where $\Gamma_{\mathrm{o}} \sim 10^{-9} \mathrm{~s}$ is an atomic-scale relaxation rate. ${ }^{10}$ In this section, we show that this relaxation is a rather poor estimate if one considers the permanent-magnet case of particles with high anisotropy.

Insertion of Eq. (5) into the transition rates $W i_{j}=\Gamma_{\mathrm{o}}$ $\exp \left(-E_{b} / k_{B} T\right)$ of the master equation $\mathrm{d} p_{\mathrm{i}} / \mathrm{d} t=\Sigma_{j}\left(W_{\mathrm{ij}} p_{\mathrm{j}}-W_{\mathrm{ji}}\right.$ $p_{\mathrm{i}}$ ) yields, with $p_{1}+p_{2}=1$ and $p_{1}-p_{2}=m$, the following familiar two-level equation of motion for the normalized magnetization:

$$
\begin{aligned}
\mathrm{d} m / \mathrm{d} t= & \Gamma_{\mathrm{o}} \exp \left[-\beta K V\left(1-H^{2} / H_{o}^{2}\right)\right] \\
& \times[\sinh (\beta h)-m \cosh (\beta h)] .
\end{aligned}
$$

Here $\beta=1 / k_{\mathrm{B}} T$ and $h=\mu_{\mathrm{o}} M H V$. For large $K$ and small $H$, this equation simplifies to

$$
\mathrm{d} m / \mathrm{d} t=\Gamma_{\mathrm{o}} \exp (-\beta K V)(\beta h-m) .
$$

However, the right-hand side of this equation depends on both $m$ and $\beta(t)$. To approximately solve Eq. (7), we first take into account that $T=\gamma t$ yields $\mathrm{d} m / \mathrm{d} t=\mathrm{d} m / \mathrm{d} T=0$ at the peak position. Both $\Gamma_{\mathrm{o}}$ and the exponential function are positive, so that the peak height $m_{\mathrm{o}}$ and its position $T_{\mathrm{o}}$ obey $k_{\mathrm{B}} T_{\mathrm{o}}=m_{\mathrm{o}} / h$. We start by formally integrating Eq. (9) from $t=0$ to $t=t_{\mathrm{o}}$ :

$$
m_{\mathrm{o}}=\Gamma_{\mathrm{o}} \int_{0}^{t_{\mathrm{o}}} \exp (-\beta K V)(\beta h-m) \mathrm{d} t .
$$

Next, we take into account that the exponent $-\beta K V$ is largest at high temperatures, that is, for $t \approx t_{\mathrm{o}}$. We can therefore write $t=t_{\mathrm{o}}-t^{\prime}$ and $T=T_{\mathrm{o}}-\gamma t^{\prime}$, expand the integrand of Eq. (9) into powers of $t^{\prime}$, and keep the linear terms only. The evaluation of the integral is then straightforward and yields

$$
m_{\mathrm{o}}=\Gamma_{\mathrm{o}} \exp (-\beta K V) T_{\mathrm{o}}^{2} h / \gamma K^{2} .
$$

Using $h=m_{\mathrm{o}} / k_{\mathrm{B}} \mathrm{T}_{\mathrm{o}}$ and dividing by $m_{\mathrm{o}}$ we obtain a selfconsistent equation for $T_{\mathrm{o}}$ :

$$
\exp \left(K V / k_{\mathrm{B}} T_{\mathrm{o}}\right)=\Gamma_{\mathrm{o}} t_{\mathrm{o}}\left(k_{\mathrm{B}} T_{\mathrm{o}} / K V\right)^{2}
$$

Without the quadratic term, this equation would reproduce the $25-k_{\mathrm{B}} T$ dependence, but $\left(k_{\mathrm{B}} T_{\mathrm{o}} / K V\right)^{2} \ll 1$ reduces the numerical factor by about $\ln \left(25^{2}\right)=6.38$. A more accurate numerical analysis of Eq. (10) shows that $25 k_{\mathrm{B}} T$ must be replaced by $19.1 k_{\mathrm{B}} T$. Physically, the effectiveness of the ZFC energy barriers is reduced, because the high anisotropy limits $m_{\mathrm{o}}$ and means that the switching of a small fraction of the particles is sufficient to realize the $\mathrm{ZFC}$ peak.

\section{CONCLUSIONS}

In summary, we have investigated some theoretical implications of experimental approaches to characterize ensembles of hard-magnetic nanoparticles. Two of the explicit examples discussed in this paper are the inequivalence of mean-field-type magnetostatic and exchange interaction fields in hysteresis loops and the replacement of the factor 25 in the analysis of ZFC curves by a reduced value of 19.1 , caused by the high anisotropy of the nanoparticles.

\section{ACKNOWLEDGMENTS}

This research is supported by DOE (RS, DJS), NSFMRSEC (TAG, XHW), ARPA-E (BB), BREM (JES), and NCMN.

${ }^{1}$ C. B. Rong, D. R. Li, V. Nandwana, N. Poudyal, Y. Ding, Z. L. Wang, H. Zeng, and J. P. Liu, Adv. Mater. 18, 2984 (2006).

${ }^{2}$ B. Balasubramanian, R. Skomski, X.-Zh. Li, Sh. R. Valloppilly, J. E. Shield, G. C. Hadjipanayis, and D. J. Sellmyer, Nano Lett. 11, 1747 (2011).

${ }^{3}$ E. Callen, Y. J. Liu, and J. R. Cullen, Phys. Rev. B 16, 263 (1977).

${ }^{4}$ R. Skomski, J. Phys.: Condens. Matter 15, R841 (2003).

${ }^{5}$ D. Stoyan and J. Mecke, Stochastische Geometrie (Akademieverlag, Berlin, 1983).

${ }^{6}$ L. Onsager, J. Am. Chem. Soc. 58, 1486 (1936).

${ }^{7}$ K.-H. Fischer and A. J. Hertz, Spin Glasses (University Press, Cambridge 1991).

${ }^{8} \mathrm{P}$. Fulde, Electron Correlations in Molecules and Solids (Springer, Berlin, 1991).

${ }^{9}$ J. S. Smart, Effective Field Theories of Magnetism (Saunders, Philadephia, 1966).

${ }^{10}$ R. Becker and W. Döring, Ferromagnetismus (Springer, Berlin, 1939). 